\title{
Predictive markers associated with dental implants biocompatibility using proteomic analysis
}

F.Romero-Gavilán ${ }^{1}$, N.Araújo-Gomes ${ }^{1}$, A.M. Sánchez-Pérez², F.Elortza ${ }^{3}$, I.Goñi ${ }^{4}$, M.Gurruchaga4 ${ }^{4}$, J.Franco ${ }^{5}$, J.Suay 1

1 Dpto. Ingeniería de Sistemas Industriales y Diseño and ${ }^{2}$ Dpto. Medicina. Universidad Jaume I (Castellón, Spain). ${ }^{3}$ Proteomics Platform CIC bioGUNE (Derio, Spain). ${ }^{4}$ Dpto. Ciencia y Tecnología de Polímeros. POLYMAT. Universidad del País Vasco (San Sebastián, Spain). ${ }^{5}$ GMI-llerimplant Group (Lleida, Spain)

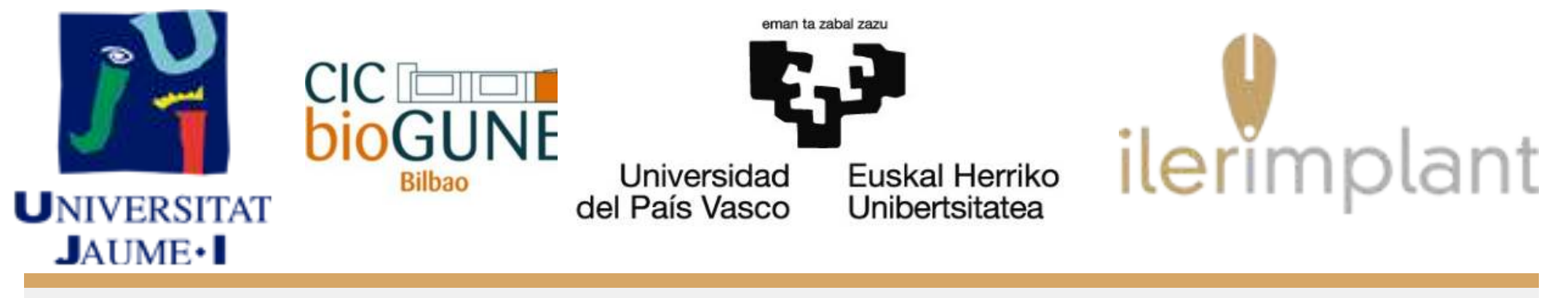

Abstract

Implants successfulness depends on their interaction with body fluids [1]. As a result, a variety of blood proteins is adhered onto the implant surface mediating subsequent biological processes. The protein layer properties will define the natural healing course by controlling processes ike blood coagulation, inflammation and immune response [2]. To study implant-related body responses, different silicon-based coatings were synthesized using the sol-gel process. As we have demonstrated in previous papers $[3,4]$, the sol-gel method is a relatively simple and inexpensive way to synthesize different coatings that can be applied directly to the implant surface, conferring the desired osteorregenerative properties. In this work we prepared various formulations of sol-gel hybrids and examined the effect of their composition on in vitro and in

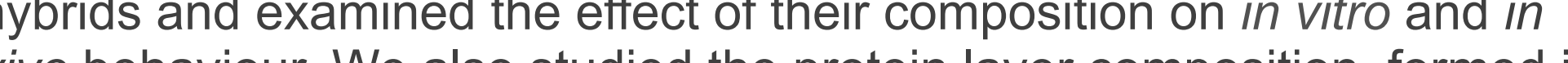
vivo behaviour. We also studied the protein layer composition, formed in each case in contact with human serum. Results confirm that chemica structure and composition of surfaces affect protein adsorption and hence biocompatibility of implants. Proteomic studies might be the key to get to know how this protein layer interacts either with biomaterials or bone regenerative process. This knowledge can be useful to assess the success or failure of an implant [5]

\section{Background and Aim}

Backgroud: In spite of the high success of dental implants, two main shortcomings, i.e. lack of osseointegration in patients with low bone quality and peri-implantitis, make the research in this field still challenging. On the one hand, patient's physiology plays a crucial role in either the immunological response or osseointegration subsequent to the insertion of dental implants. On the other, new Ti surface treatments or other materials developments carry out in vitro and in vivo assays that, in many cases, show a lack of correlation between results.

- Aim/Hypothesis: Blood protein deposition onto the biomaterial surface occurs immediately after implantation. Thus, our hypothesis is that it might be possible to predict the final in vivo outcome by the identification of quantity and type of proteins adsorbed onto the implant surface using proteomic analysis. Thus, a correlation between in vivo and proteomics results should be possible. Results obtained with various coatings designed to enhance osseointegration will be discussed.

\section{Methods and Materials}

- Four distinct hybrid sol-gel materials were synthesized employing as precursors methyltrimethoxysilane (M), 3glycidoxypropyl-trimethoxysilane $(\mathbf{G})$, tetraethyl orthosilicate (T) and triethoxyvinylsilane $(\mathbf{V})$ in molar ratios of $70 \mathrm{M}: 30 \mathrm{~T}$, 35M:35G:30T, 50M:50G and 50V:50G.

- SLA-Ti discs were coated with prepared materials by dipcoating. In vitro biomaterial evaluation was carried out with MC3T3-E1 cells. In vivo experimentation was carried out implanting sol-gel coated Ti dental screws (Ilerimplant - GMI Frontier model $($ ) by dip-coating in rabbit proximal tibia. Animals were euthanized two weeks after implantation.

- Proteomic assay was conducted by incubating the discs with human blood serum for $3 \mathrm{~h}$. Non-adhered proteins were removed with 5 consecutive washes of $\mathrm{ddH}_{2} \mathrm{O}$ and one wash of saline solution. Proteins adsorbed to each surface were eluted with SDS-DTT solution. The identification and quantification of eluted proteins was performed using mass spectrometry (LC/MS/MS).

\section{Acknowledgements}

This work was supported by Spanish Ministry of Economy and Competitiveness through "MAT 2014-51918-C2-2-R", Universidad Jaume I with "P11B2014-19" and "Predoc/2014/25", Generalitat Valenciana "Grisolia/2014/016", the University of the Basque Country (UPV/EHU) through "UFI11/56" and Basque Government through the program for Grupos consolidados IT-611-13 and Ilerimplant S.L.

\section{Results}

Despite their physico-chemical differences, almost all the tested coatings showed good in vitro behaviour. Non-statistical in vitro differences were found.

- Regardless of these results, the in vivo experimentation displayed the formation of an immune structure, a layer of fibrous connective tissue, surrounding 50M:50G and 50V:50G coatings. However, 70M:30T and 35M:35G:30T achieved a good osseointegration for this period (fig.1)
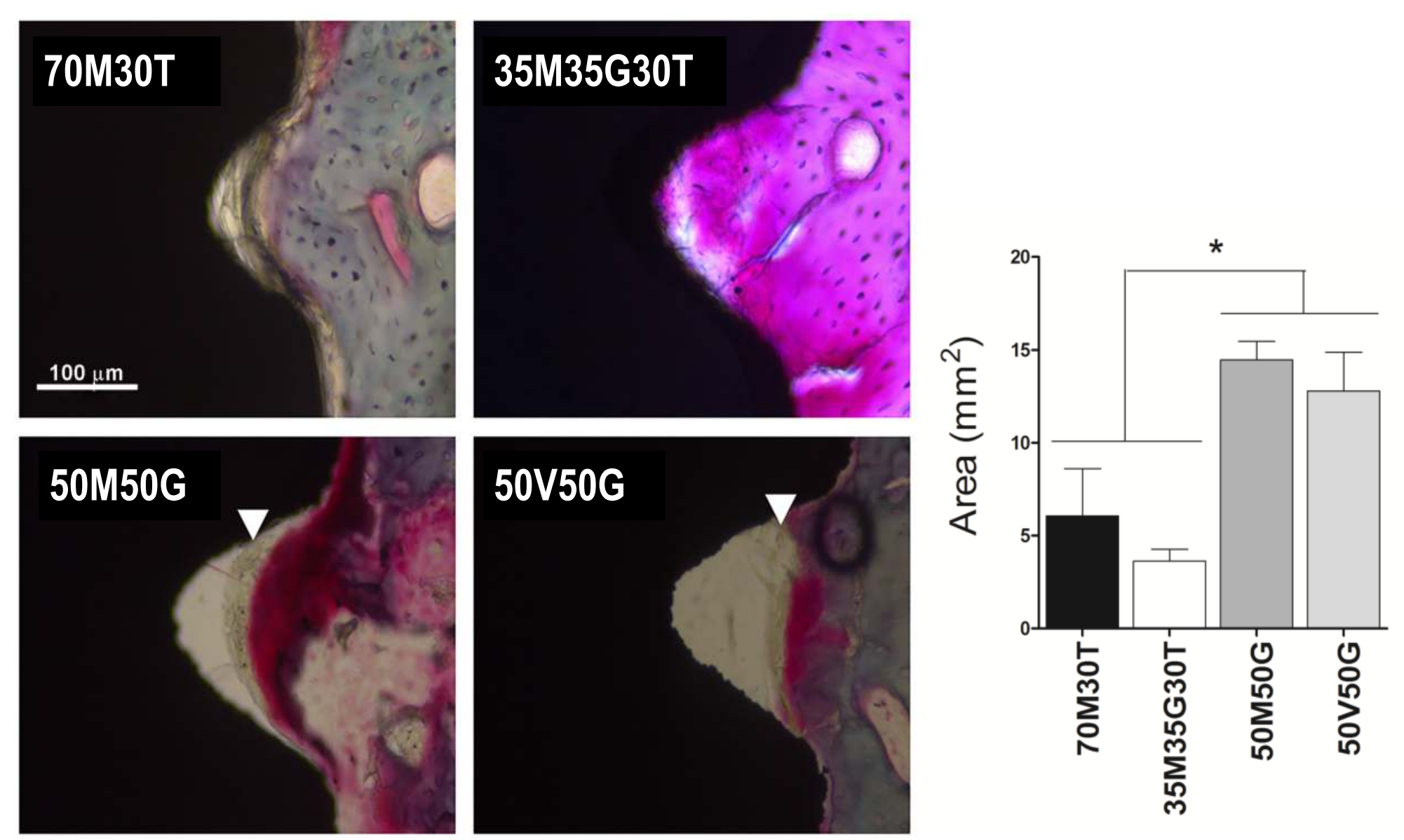

Figure 1: Light microscopy images of histological samples after 2 weeks and respective quantification of the area $\left(\mathrm{mm}^{2}\right)$ occupied by fibrous connective tissue (Anova $p$-value $<0.05, n=3$ )

- The proteomic analysis identified 171 distinct proteins adhered to the coatings [6]. Comparison of the protein profiles of coatings with different in vivo behaviour, resulted on the identification of a group of 9 proteins significantly more adsorbed to the biomaterials with poor biocompatibility, including proteins like CRP, SAMP, C1QC or C1s. Interestingly, all of them have a direct or indirect role on the complement pathway. In fact, a great part of them belong to a protein cluster related to acute inflammatory processes (fig. 2).

Inflammatory/lmmune response

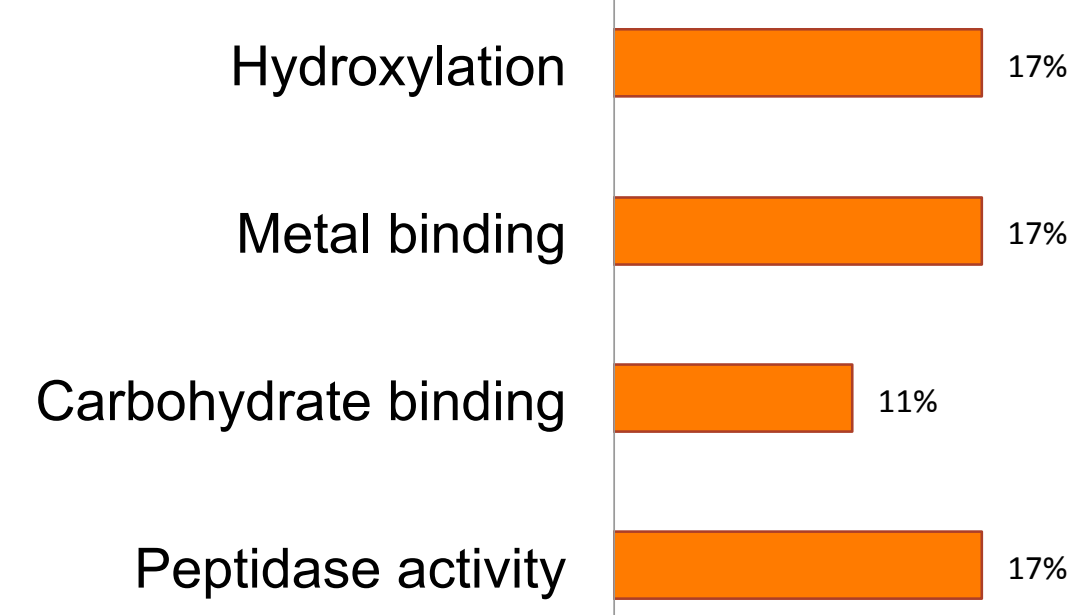

Figure 2: Function of proteins adsorbed differentially on materials with bad biocompatibility (Anova p-value $<0.05, n=4$ ).

\section{Conclusions}

- The results suggest that the different proteomic layer composition of biomaterials with bad in vivo outcome could explain the fibrous connective tissue formation.

- A cluster of possible biomarkers related with biocompatibility problems have been identified. Therefore, proteomic assays could represent an important tool for the prediction of dental implants biocompatibility.

\section{References}

Puleo DA, Nanci A. Biomaterials. 1999; 20:2311-21

2. Brash JL, Ten Hove P. Thromb. Haemost. 1984; 51:326-30

Martínez-Ibáñez M, Juan-Díaz MJ, Lara-Saez I, Coso A, Franco J, Gurruchaga M, Goñi I. Journal of Materials Science: Materials in Medicine. 2016; 27(4): 1-9 Juan-Díaz, MJ, Martínez-lbáñez M, Lara-Sáez I, da Silva S, Izquierdo, R,

Horbett TA. Biomater. Sci. (Third Ed). 2013; p. 394-408 6. Romero-Gavilán F, Sánchez-Pérez AM, Araújo-Gomes N, Azkargorta M, lloro I,
Elortza- F, Gurruchaga M, Goñi I, Suay J. Biofouling 2017; DOI: 10.1080/08927014.
2017.1356289. 